\title{
Implications of four-dimensional weather cubes for improved cloud-free line-of-sight assessments of free-space optical communications link performance
}

\author{
Steven T. Fiorino, ${ }^{\text {a,* }}$ Santasri R. Bose-Pillai, ${ }^{\text {a,b }}$ Jaclyn Schmidt, ${ }^{\text {a,b }}$ \\ Brannon Elmore, ${ }^{\text {a,b }}$ and Kevin Keefer ${ }^{\mathrm{a}, \mathrm{b}}$ \\ ${ }^{a}$ Air Force Institute of Technology, Department of Engineering Physics, Wright-Patterson AFB, \\ Ohio, United States \\ ${ }^{\mathrm{b}}$ Applied Research Solutions, Beavercreek, Ohio, United States
}

\begin{abstract}
We advance the benefits of previously reported four-dimensional (4-D) weather cubes toward the creation of high-fidelity cloud-free line-of-sight (CFLOS) beam propagation for realistic assessment of autotracked/dynamically routed free-space optical (FSO) communication datalink concepts. The weather cubes accrue parameterization of optical effects and custom atmospheric resolution through implementation of numerical weather prediction data in the Laser Environmental Effects Definition and Reference atmospheric characterization and radiative transfer code. 4-D weather cube analyses have recently been expanded to accurately assess system performance (probabilistic climatologies and performance forecasts) at any wavelength/frequency or spectral band in the absence of field tests and employment data. The 4-D weather cubes initialize an engineering propagation code, which provides the basis for comparative percentile performance binning of FSO communication bit error rates (BERs) as a function of wide-ranging azimuth/elevation, earth-to-space uplinks. The aggregated, comparative BER binning analyzes for different regions, times of day, and seasons applying a full year of 4-D weather cubes data provided numerous occasions of clouds, fogs, and precipitation events. The analysis demonstrated the utility of 4-D weather cubes for adroit management of CFLOS opportunities to enhance performance analyses of point-to-point as well as evolving multilayer wireless network concepts. () The Authors. Published by SPIE under a Creative Commons Attribution 4.0 Unported License. Distribution or reproduction of this work in whole or in part requires full attribution of the original publication, including its DOI. [DOI: 10.1117/1.OE.59.8 $.081808]$
\end{abstract}

Keywords: free-space optical communications; cloud-free line-of-sight; laser propagation; turbulence; atmospheric characterization; weather cubes.

Paper 191795SS received Dec. 24, 2019; accepted for publication Jun. 23, 2020; published online Jul. 17, 2020.

\section{Introduction}

The lure of terrestrial free-space optical (FSO) communications architectures to meet the evergrowing demand for increased bandwidth and data rates has motivated dozens if not hundreds of research teams the world over for more than two decades. This enthusiasm is offset to some extent by light's known susceptibility to atmospheric effects, especially optical turbulence and extinction due to aerosols, clouds, fog, and precipitation. Optical turbulence effects have received special attention. These arise from ever-present random changes in the ambient air's refractivity along the path of one's communication channel and include beam spreading and irradiance fluctuations otherwise characterized as scintillation. The index of refraction structure constant $C_{n}^{2}$ is widely used to classify optical turbulence strength. In turn, this parameter is folded into the calculation of a scintillation index. This and other measured or calculated system parameters, such as irradiance at the receiver, enable teams to evaluate and/or predict a link's bit error rate (BER) performance. Barrios and Dios ${ }^{1}$ compiled an excellent summary of this methodology.

*Address all correspondence to Steven T. Fiorino, E-mail: steven.fiorino@afit.edu 
Naturally, assessing and even predicting $C_{n}^{2}$ along one's communication channel has captured great interest. Although all can claim familiarity with scintillometers, new equipment and techniques relying, for example, on time-lapse imagery and weather radar have been advanced to determine local $C_{n}^{2}{ }^{2-4}$ Though not predictive, such measures can provide real-time insight on likely link performance. With an eye on first-principles models such as those of Tatarskii, Thiermann-Kohnle, and Monin-Obhukov, others have relied on traditional scintillometry and extensive linear regression techniques to derive $C_{n}^{2}$ as a function of standard meteorological parameters such as wind speed, temperature, relative humidity, and solar flux. ${ }^{5-9}$ These relationships were expressed in alternate forms to account for time of day and/or solar flux, season, and even surface cover. To the extent that these relationships relied solely on standard meteorological parameters found in weather forecasts, $C_{n}^{2}$ prediction was suggested..$^{10}$ Recently, efforts have focused on enhancing correlations of commonly measured meteorological parameters with $C_{n}^{2}$ irrespective of season, climate, and topography while also utilizing actual FSO links, albeit of relatively short point-to-point extent, to infer the $C_{n}^{2}{ }^{11,12}$

As for the other primary influence on FSO performance, atmospheric constituentsmolecules, aerosols, water droplets, and ice crystals-in aggregate diminish laser irradiance at the link's receiver according to Beer's law. For FSO operating wavelengths, light scatter is the predominant source of extinction. Kim et al. provided a comprehensive survey of such effects relative to these constituents. ${ }^{13}$ Though many times extinction is considered in terms of link margins, it also can be factored into consideration of a link performance relative to BER. This will be shown in the following section.

In this work, numerical weather prediction (NWP) data produced by the National Oceanic and Atmospheric Administration's (NOAA's) Global Forecast System (GFS) model are interrogated and aggregated using the Laser Environmental Effects Definition and Reference (LEEDR) model to produce gridded four-dimensional (4-D) weather cubes. ${ }^{14,15}$ The weather cubes retain the features associated with the GFS numerical weather data, both spatial and temporal, which, in turn, enables definition of realistic cloud and rain events and cloud-free line-ofsight (CFLOS) assessments. Intrinsically, a verified and validated atmospheric characterization and radiative transfer code, LEEDR, uses an embedded modified Tatarskii turbulence profile calculator so the 4-D weather cube concurrently presents vertical and horizontal $C_{n}^{2}$ profiles from the NWP data. LEEDR also contains both the worldwide global aerosol dataset aerosol climatology, a first-principles rain and cloud physical model, and a full Mie scattering calculator to comprehensively treat extinction effects. As such, this work evaluates the 4-D weather cube proficiency for characterizing the highly uniform and/or nonuniform state of the atmosphere and associated optical effects with high fidelity from transmitter to receiver. Furthermore, the 4-D weather cube method offers the prospect of both forecasting FSO link performance, and if needed, forensically evaluating the reasons for link dropouts. The immediate implications for efficiently supplementing an ultrahigh bandwidth and data rate communications FSO network with alternate, but lesser bit rate capabilities, such as 10-GHz microwave (MW) links, are readily apparent. Beyond that the 4-D weather cube's predictive and better resolved intrapath atmospheric effects capabilities lend themselves to a recent uptick in applying machine learning to advance cost-effective concepts for hard switching between FSO and MW links when anticipated performance thresholds are crossed. ${ }^{16}$

The next section on tools and methods discusses the 4-D weather cubes, their utility, and the approach taken to create them, including LEEDR's principle role. As to the cube creation step, a short summary is also included describing the principal computational node-level interrogation and aggregation technique that were used. The second portion of the tools and methods section outlines the development of the prototype BER performance metric around which the trade space percentile performance binning was organized. In addition, the discussion includes key assumptions that were applied to enable the outputs of the described HighEnergy Laser End-to-End Operational Simulation (HELEEOS) laser propagation performance code to be relevant to the BER calculation. Results from the multidimensional-as in multiple azimuth and zenith-full year at four times a day BER performance binning and CFLOS analysis are shown in Sec. 3. A brief summary of observations and conclusion are given in Sec. 4. 


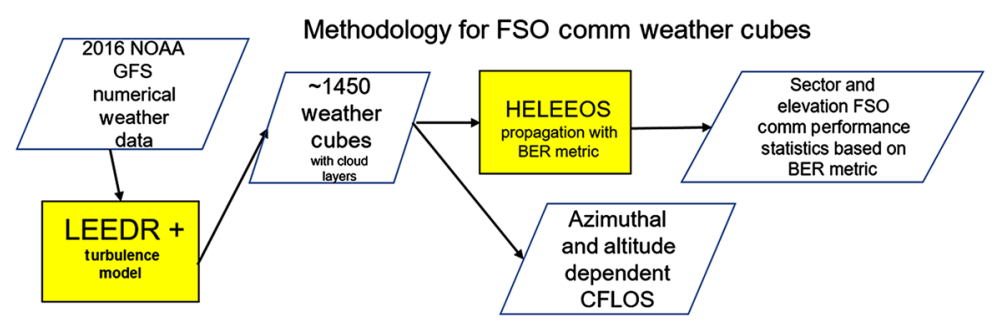

Fig. 1 A flowchart of how the FSO communications BER and CFLOS statistics were compiled using weather cubes.

\section{Analytical Tools and Methods}

This section describes the analytical tools and anchor codes used for a performance trade study of laser free-space communications, which may be a key node in future integrated kinetic and directed energy weapons architectures. Large, computationally intensive 4-D data arrays, called weather cubes, define meteorological and multispectral atmospheric effects data for volumes specific to a universal time reference, locations of interest (i.e., georeferenced light source and remote sensor/target) and a user-specified output parameter such as transmission, thus providing quantification of realistic atmospheric environmental effects. Weather cubes are anchored to LEEDR radiative transfer (extinction, transmission, and radiance) with NWP model pressure, temperature, humidity, and wind vector volumetric definitions. High-performance computing (HPC) is leveraged to efficiently generate one full year of weather cubes, consisting of 2016 NWP short-term $(6 \mathrm{~h})$ forecasts with LEEDR radiative transfer data at four times a day. Performance statistics are generated when weather cubes are coupled with a propagation code (i.e., HELEEOS in this study), allowing for performance binning and percentiles analyses for a variety of engagements that consider spatial and temporal variations in atmospheric conditions. Figure 1 shows a flowchart overview of the models and inputs used to allow the weather cube analysis of FSO communications performance and effects of CFLOS.

\subsection{Input Models}

The LEEDR code exports first principles atmospheric characterizations for directed energy weapon and electro-optical and infrared simulation codes, military, or U.S. Department of Defense (DoD) mission planners, as well as nonmilitary scientific research such as climate change impact studies. ${ }^{15}$ This fast-calculating, worldwide, surface-to- $100 \mathrm{~km}$, atmospheric characterization, and radiative transfer code has been verified and validated. Atmospheric effects are calculated for any ultraviolet and radio frequency wavelengths ( $200 \mathrm{~nm}$ to $8.6 \mathrm{~m}$ ). Using internally consistent line-by-line and correlated- $k$ distribution radiative transfer algorithms, LEEDR is capable of assessing path transmittance as well as path radiance and celestial effects on sensed signals of interest for any three-dimensional (3-D) observer/target geometry on a spherical earth. ${ }^{17}$

The NWP data incorporated into LEEDR originate from correlated, gridded real-time atmospheric analysis based on thousands of global observations, as well as weather forecasts or past reanalysis data. This feature enables postevent, nowcast, and forecast analyses of atmospheric and radiative effects for real-world scenarios and significantly improves its capabilities to perform predictive and analytical assessments in terms of trade space studies and the development of climatological databases. The previously mentioned GFS NWP model is a global, hydrostatic, operational numerical weather forecast model that is generated four times per day $(0000,0600$, 1200 , and 1800 UTC) and provides forecasts out to $384 \mathrm{~h}$ from the forecast cycle time. Meteorological data are available at 64 atmospheric levels, extending from the surface to $\sim 30 \mathrm{~km}$ in altitude. GFS data with a $45-\mathrm{km}$ horizontal grid spacing (0.5-deg resolution) are used for this study. GFS model data are made available to the public, free of cost, through NOAA's Operational Model Archive Distribution System. ${ }^{18}$

The NWP data provide the 3-D temperature and wind data LEEDR used for calculation of the refractive index structure function $C_{n}^{2}$ through the temperature structure function $C_{T}^{2}$. Using expressions derived by Tatarskii, ${ }^{5} C_{T}^{2}$ and $C_{n}^{2}$ at optical wavelengths can be determined by 


$$
\begin{gathered}
C_{n}^{2}=2.8 \frac{K_{H}}{K_{M}}\left(79 \times 10^{-6} \frac{P}{T^{2}}\right)^{2} L_{0}^{4 / 3}\left(\frac{\partial T}{\partial z}+\gamma_{d}\right)^{2}, \\
C_{T}^{2}=2.8 \frac{K_{H}}{K_{M}} L_{0}^{4 / 3}\left(\frac{\partial T}{\partial z}+\gamma_{d}\right)^{2} .
\end{gathered}
$$

The outer scale length $L_{o}$ is the largest scale of the atmospheric turbulence and is estimated to be $100 \mathrm{~m}$ in the Tatarskii calculation in LEEDR. $K_{H}$ is the eddy diffusivity for heat and $K_{M}$ is the eddy diffusivity for momentum. The ratio of these eddy transfer coefficients $K_{H} / K_{M}$ is described by Kondo et al. ${ }^{19}$ as

$$
\frac{K_{H}}{K_{M}}=\left\{\begin{array}{ll}
\frac{1}{7 R i} & \text { for } R i>1 \\
\frac{1}{6.873 R i+\frac{1}{1+6.873 R i}} & \text { for } 0.01<R i \leq 1
\end{array} .\right.
$$

The gradient Richardson number $R i$ indicates the dynamic stability of the atmosphere and gives insight into turbulence production. It can be approximated from the vertical gradients of wind and potential temperature ${ }^{20}$

$$
R i=\left(\frac{g}{\bar{T}_{v}} \frac{\partial \bar{\theta}_{v}}{\partial z}\right) /\left[\left(\frac{\partial \bar{u}}{\partial z}\right)^{2}+\left(\frac{\partial \bar{v}}{\partial z}\right)^{2}\right]
$$

where $T_{v}$ is the virtual temperature, $\theta_{v}$ is the virtual potential temperature, and $z$ is the geometric height. Alliss and Felton used this modified Tatarskii formulation and the weather research and forecast NWP output to generate a $C_{n}^{2}$ climatology over the Hawaiian Islands in 2009. ${ }^{21}$ They found, as others have as well, that this modified Tatarskii method when applied to NWP data yields realistic profiles of $C_{n}^{2}$ as the NWP data rarely, as with actual observed conditions, become perfectly adiabatic as occurs in idealized atmospheric layers. Furthermore, the $K_{H} / K_{M}$ term tends to be large (near 1.0) when the lapse of the atmosphere is near adiabatic or neutrally buoyant, and orders of magnitude smaller when the atmosphere is stable and the temperature lapse is greatly different than dry adiabatic $\left(-9.8 \mathrm{~K} \mathrm{~km}^{-1}\right)$.

\subsection{Weather Cubes}

Weather cubes are the product of the coupling of LEEDR and NWP for a region of interest, consisting of numerous vertical profiles in a mesh-file format. These large volumes provide analytical, visualization, and decision aid tools, which accurately convey multispectral (UV through rf) propagation and atmospheric effects that are associated with a location, date, and time. The primary benefit of weather cubes is that realistic multidirectional variations of atmospheric parameters based on weather conditions that may have or actually did occur are captured. The weather cubes can convey a large assortment of atmospheric parameters and 4-D variability including but not limited to temperature, pressure, relative humidity, dew point, atmospheric density, wind vectors, vertical velocity, cloud and precipitation types and locations, visible refractive index gradients $\left(C_{n}^{2}\right)$, extinction due to molecular, particulate, and hydrometeor absorption and scattering, single scattering albedo, and phase functions. The weather cubes extend from the surface to $100 \mathrm{~km}$ with a vertical resolution of $100 \mathrm{~m}$ for any number of user-defined wavelengths. As detailed earlier, turbulence effects in this study are characterized by modified Tatarskii calculations, which create realistic vertical profiles of $C_{n}^{2}$ based on NWP variables. As is to be expected, full Mie calculations are the basis for weather cube extinction. Definition of clouds and precipitation events in weather cubes is described in the following paragraph. Parametric outputs are stored in a matrix indexed by latitude, longitude, altitude, date, and time.

Although the GFS does not explicitly contain cloud and precipitation information, simple yet physically based weather placement algorithms have been implemented into weather cube data processing scripts to incorporate GFS-inferred cloud and precipitation layers for realistic sky characterizations. Recent upgrades to the algorithms now consider ice particles as well. The following GFS output variables are used to determine cloud and precipitation type, 


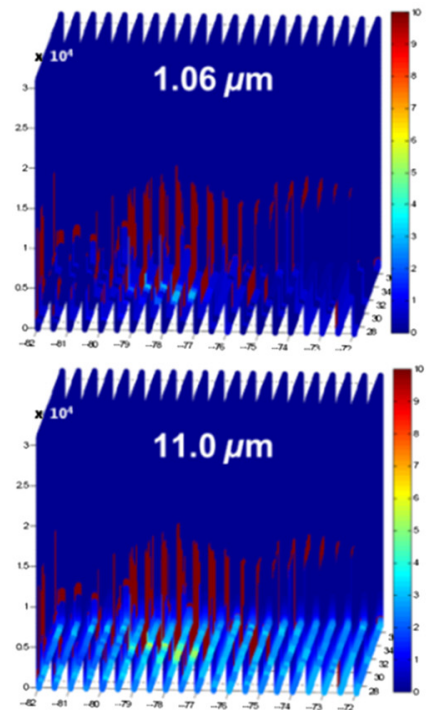

(a)

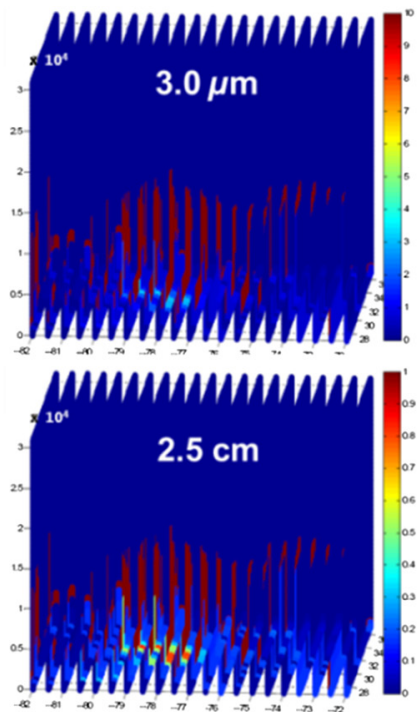

(b)

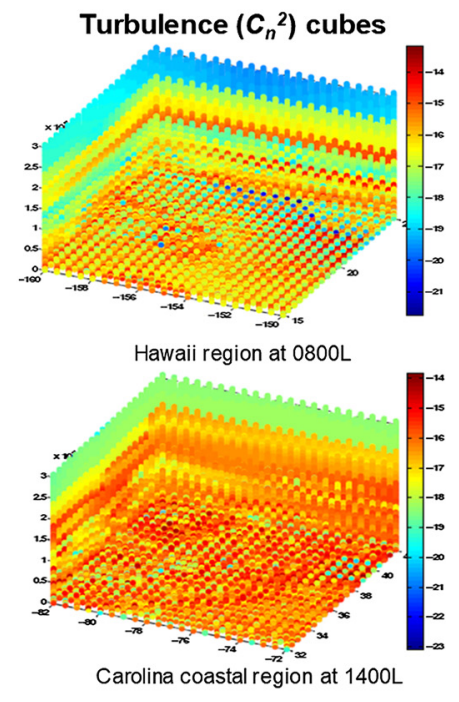

(c)

Fig. 2 (a), (b) Weather cubes depicting multispectral, atmospheric extinction for a significant weather occurrence, Hurricane Arthur on July 3, 2014, 1800 UTC. Maroon color indicates the location of clouds (high extinction values) at all 4 wavelengths, but note the change in extinction scale at $2.5 \mathrm{~cm}$ to showcase the location of rain fields located near the surface. (c) Weather cubes depicting $C_{n}^{2}$ data calculated using the modified Tatarskii method and NWP data from the GFS model on August 18, 2016, at 1800 UTC.

including base and top heights: relative humidity, vertical velocity (Lagrangian rate of change of pressure with time in units of Pascals per second), temperature, and 3-h surface precipitation total. Cloud types are assigned as either fog, stratus, cumulus, cirrus, or mixed-phase clouds. Precipitation fields are determined if the following two parameters are met: a cloud is present and a 3-h surface precipitation total $>0$ in. is derived from the model. The placement of precipitation fields, with a designated precipitation rate based on the 3-h surface precipitation total, begins at the middle of the cloud and extends to the surface of the Earth. Temperature defines the type of precipitation, snow, or rain. A validation study was conducted in 2016 on the sky characterization algorithms using NASA's Moderate Resolution Imaging Spectroradiometer (MODIS) to assess cloud fields and Next Generation Radar (NEXRAD) data from the Wilmington, North Carolina (KTLX) site to assess rain field placements. The case study was focused on Hurricane Arthur, a well-organized meteorological phenomenon that made landfall near Wilmington, North Carolina, in July 2014. The resulting cloud and precipitation fields were comparable to both MODIS and NEXRAD data. More information on the validation results are available. ${ }^{15}$

A representation of weather cubes depicting atmospheric extinction (absorption and scattering, units $=\mathrm{km}^{-1}$ ) at four wavelengths for a significant weather case, Hurricane Arthur on July 3, 2014, at 1800 UTC, is shown in Fig. 2. The weather cubes used for Fig. 2 contain meteorological information and multispectral atmospheric effects for 36 wavelengths of interest, but any number of wavelengths within the spectral range of $200 \mathrm{~nm}$ to $8.6 \mathrm{~m}$ can be included in weather cubes. The maroon color-indicative of high extinction values-points to the presence of clouds at all four wavelengths. The midwave infrared band is highly attenuated by water vapor; note that low-level water vapor effects, seen as the bright blue and green colors $\left(3\right.$ to $\left.6 \mathrm{~km}^{-1}\right)$, are evident near the surface of the $11.0-\mu \mathrm{m}$ extinction cube. The extinction scale was reduced to a maximum of $1 \mathrm{~km}^{-1}$ for the $2.5-\mathrm{cm}$ wavelength graphic to showcase the location of rain fields near the surface, as rain attenuation is the dominant feature of MW radio frequencies. Figure 2 also displays weather cubes with volumetric $C_{n}^{2}$ profile data calculated using the modified Tatarskii method in LEEDR and NWP data from the GFS model August 18, 2016, at 1800 UTC.

The development of weather cubes was motivated by the need to better render atmospheric effects when assessing adversary threats for military gaming simulations. But this innovative tool 
goes far beyond advancing war-gaming simulations, opening doors to furthering remote sensing, directed energy performance assessments, and climate research. ${ }^{18}$ When weather cubes are fully integrated with propagation performance codes, such as HELEEOS, statistics can be derived by assessing performance results for a given engagement in various atmospheric conditions. HELEEOS is a fast-running, scaling law code that is comprised of the following three codes that provide robust, time efficient solutions compared to computationally expensive, firstprinciples wave optics models: (1) LEEDR defines the atmospheric characterization and radiative transfer effects; (2) scaling for HEL and relay engagement developed by MZA Associates Corporation produces directed energy propagation metrics and irradiance outputs; and (3) the SAIC-Nutronics developed adaptive optics compensation of the thermal blooming wave optics model, which enables higher fidelity representation of non-Gaussian thermal blooming effects, nonlinear interactions with turbulence, as well as instabilities associated with employing one's beam control/adaptive optics subsystem. ${ }^{15}$ Since LEEDR is fully integrated into HELEEOS, the HELEEOS-weather cube interface is straightforward thus providing for full-dimensional consideration of atmospheric variability and effects for any laser propagation scenario.

\subsection{Prototype BER-Based Laser Communications Performance Metric}

A performance metric is required to evaluate the plausibility of using free-space laser communications, also referred to FSO communications. Laser performance metrics generally consider total transmitted power to the target in the form of peak irradiance or power delivered in a predetermined "bucket" size, but the chosen metric for the present work is BER. This is partly because communication links are primarily concerned with the quality of the signal and the relative success of information delivery, transmitted between two points via laser energy rather than delivering sufficient energy to damage a target. However, a BER performance metric might yield additional important information about the environmental effects along a propagation path at BERs deemed acceptable or unacceptable for any communication link whether FSO, rf, or a hybrid. Though BERs that preclude effective communication would typically be ignored, predictive tools to both investigate underlying environmental contributions and optimize communication networks a day to weeks in advance are of interest. Thus, the goal in this present research is to develop and evaluate a new predictive tool-4-D weather cubes-using a prototype BER metric, not necessarily simulate and evaluate the feasibility of an FSO communications uplink engineering design framework.

Using HELEEOS and weather cubes, an uplink scenario was considered between a ground station and a low Earth orbit (LEO) satellite at an altitude of $250 \mathrm{~km}$. Following standard practice, assuming transmission of "0"s and " 1 "s is equally probable, a BER is defined as

$$
\mathrm{BER}=\frac{1}{2}[P(0 \mid 1)+P(1 \mid 0)],
$$

where $P()$ are the conditional probabilities and indicate the probability that a " 0 " is received given a " 1 " is transmitted and a probability that a " 1 " is received given a " 0 " is transmitted, respectively. The BER depends on the received signal level and the receiver noise level. The simplest signaling format is the On-off keying where a binary " 1 " is represented by a pulse and a binary " 0 " represents the absence of a pulse. When a pulse is transmitted, the receiver detects optical power corresponding to the signal level and the background. In the absence of a pulse, the receiver receives only background radiation. The receiver has a threshold detector to detect which symbol is transmitted. Assuming Gaussian statistics and an avalanche photodiode (APD) as a receiver, the average current and associated noise current generated at the output of the APD when a pulse is transmitted are given by the following equations:

$$
\begin{gathered}
\mu_{1}=e G \frac{\eta}{h \nu}\left(P_{s}+P_{\mathrm{BG}}\right)+i_{D}, \\
\sigma_{1}^{2}=2 B\left[\frac{e^{2} \eta}{h \nu} F G^{2}\left(P_{s}+P_{\mathrm{BG}}\right)+\frac{2 K T}{R_{L}}+\sigma_{D}^{2}\right],
\end{gathered}
$$




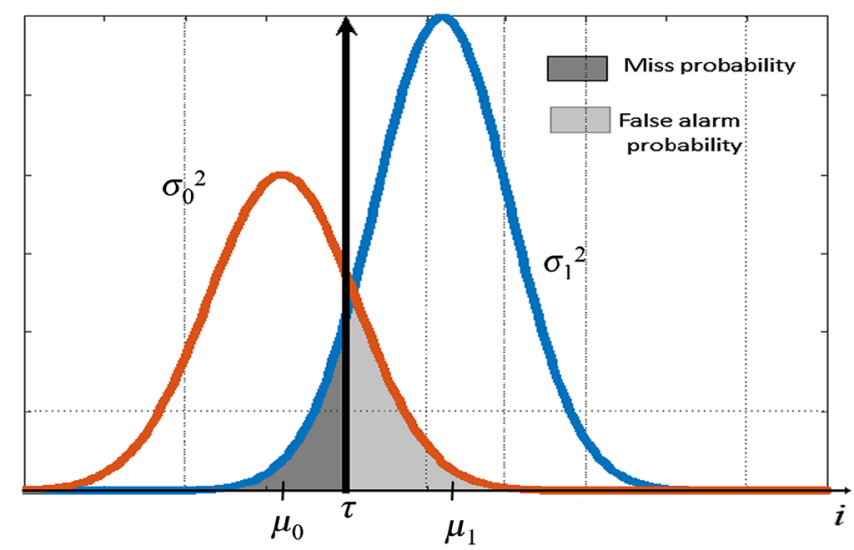

Fig. 3 Receiver output distribution under Gaussian noise assumptions.

where $e$ denotes the charge of an electron, $G$ is the intrinsic gain of the photodetector, $\eta$ is the quantum efficiency, $h$ is Planck's constant, $v$ is the optical frequency, $P_{s}$ is the received signal optical power, $P_{\mathrm{BG}}$ is the background optical power, $i_{D}$ is the dark current of the photodetector, $F$ is the excess noise factor, $K$ is the Boltzmann constant, $T$ is the operating temperature, $B$ is the electrical bandwidth, $R_{L}$ is the load resistor, and $\sigma_{D}^{2}$ is the dark current noise. Similarly, the average current and associated noise current generated when no pulse is transmitted are

$$
\begin{gathered}
\mu_{0}=e G \frac{\eta}{h \nu} P_{\mathrm{BG}}+i_{D}, \\
\sigma_{0}^{2}=2 B\left[\frac{e^{2} \eta}{h \nu} F G^{2} P_{\mathrm{BG}}+\frac{2 K T}{R_{L}}+\sigma_{D}^{2}\right] .
\end{gathered}
$$

Figure 3 shows the probability density function for the cases of a pulse being transmitted $\left(\sigma_{1}^{2}\right)$ and no pulse being transmitted $\left(\sigma_{0}^{2}\right)$. If the receiver output is higher than the threshold when no pulse is transmitted, a false alarm is generated, as seen by the light gray shading under the $\sigma_{1}^{2}$ curve. On the other hand, if the receiver output is lower than the threshold, when a pulse is transmitted, it is a miss as indicated by the dark gray shading under the $\sigma_{0}^{2}$ curve. Hence the probability of false alarm is

$$
P(1 \mid 0)=Q\left(\frac{\tau-\mu_{0}}{\sigma_{0}}\right)
$$

where $Q(x)=\frac{1}{\sqrt{2 \pi}} \int_{x}^{\infty} e^{-x^{2} / 2} \mathrm{~d} x$ is the Gaussian $Q$-function and $\tau$ is the threshold. Similarly, the probability of miss is

$$
P(0 \mid 1)=Q\left(\frac{\mu_{1}-\tau}{\sigma_{1}}\right)
$$

In the present analysis, the threshold was taken to be half of the received signal.

In the presence of optical turbulence, the received irradiance fluctuates randomly due to scintillation. Hence, the BER in Eq. (5) needs to be averaged over all possible optical signal levels according to the statistical distribution of the received irradiance. The distribution model for received irradiance considered in this study was the gamma-gamma distribution given by ${ }^{22}$

$$
p_{I}(I)=\frac{2(\alpha \beta)^{(\alpha+\beta) / 2}}{\Gamma(\alpha) \Gamma(\beta) I}\left(\frac{I}{\langle I\rangle}\right)^{(\alpha+\beta) / 2} K_{\alpha-\beta}\left(2 \sqrt{\frac{\alpha \beta I}{\langle I\rangle}}\right),
$$

where $\Gamma()$ is the gamma function, $\langle I\rangle$ is the mean irradiance, $K_{n}(x)$ is the modified Bessel function of the second kind and order $n$, and $\alpha$ and $\beta$ are the parameters related to large scale and small scale scintillation, respectively, 
Fiorino et al.: Implications of four-dimensional weather cubes...

$$
\begin{gathered}
\alpha=\frac{1}{\exp \left\{\frac{0.49 \sigma_{I}^{2}}{\left[1+0.18 d^{2}+0.56\left(2+L / F^{\prime}\right) \sigma_{I}^{12 / 5}\right]^{7 / 6}}\right\}-1}, \\
\beta=\frac{1}{\exp \left[\frac{0.51 \sigma_{I}^{2}\left(1+0.69 \sigma_{I}^{12 / 5}\right)^{-5 / 6}}{1+0.90 d^{2}+0.62 d^{2} \sigma_{I}^{12 / 5}}\right]-1} .
\end{gathered}
$$

Here, it is assumed that perfect tracking is implemented. In Eqs. (13) and (14), $F^{\prime}$ is the phase front radius of curvature of the beam at the receiver plane, $L$ is the path length, and $\sigma_{I}^{2}$ is the on-axis scintillation index of the beam under weak fluctuation. The scintillation index for the beam under weak fluctuation was obtained by referencing the log-amplitude variance calculated by HELEEOS, and

$$
d=\sqrt{\frac{k D^{2}}{4 L}},
$$

where $D$ is the receiver aperture diameter and $k$ is the wavenumber. The above model of received irradiance works for all levels of turbulence.

The BER in the presence of scintillation is thus ${ }^{1}$

$$
\operatorname{BER}=\int_{0}^{\infty} \operatorname{BER}(I) p_{I}(I) \mathrm{d} I .
$$

The mean irradiance $\langle I\rangle$ was obtained from HELEEOS as well. The background optical power was obtained from the path radiance value from LEEDR

$$
P_{\mathrm{BG}}=L_{B} A(\mathrm{FOV})^{2} \Delta \lambda
$$

where $L_{B}$ is the path radiance for the wavelength considered, $A$ is the area of the receiving aperture, FOV is the field of view of the receiving aperture, and $\Delta \lambda$ is the bandwidth of the optical filter at the receiver.

The FSO system parameters used in the present work were selected to ensure some quantifiable amount of modeled propagating laser energy would reach a simulated detector in space in any weather conditions, even thick cloudiness. These system parameters are shown in Table 1 and are not representative of any practical FSO communications system. Although the bandwidth of $1.5 \mathrm{GHz}$ is not necessarily an unreasonable value for an FSO system, more typically this bandwidth would be used in a system with a telescope no larger than $30 \mathrm{~cm}$ in diameter and a transmitting power of no more than a $\sim 1 \mathrm{~W} .{ }^{22}$ Furthermore, the $\Delta \lambda$ optical filter size of $630 \mathrm{~nm}$ is

Table 1 Parameters used in performance study.

\begin{tabular}{lccc}
\hline \hline Simulation parameter & Value & Simulation parameter & Value \\
\hline$\lambda$ & $1.55 \mu \mathrm{m}$ & $R_{L}$ & $50 \mathrm{ohms}$ \\
$G$ & 10 & $\sigma_{D}^{2}$ & $0.0225 \mathrm{pA}$ \\
$\eta$ & 0.8 & $D$ & $2.5 \mathrm{~cm}$ \\
$i_{D}$ & $50 \mathrm{nA}$ & Receiver height & $250 \mathrm{~km}$ \\
$B$ & $1.5 \mathrm{GHz}$ & FOV & $0.12 \mathrm{rad}$ \\
$F$ & 3.2 & $\Delta \lambda$ & $630 \mathrm{~nm}$ \\
$T$ & $298 \mathrm{~K}$ & Transmitting aperture diameter & $1 \mathrm{~m}$ \\
$T X$ & $100 \mathrm{~W}$ & & \\
\hline \hline
\end{tabular}


excessively large and should be closer to $\sim 10 \mathrm{~nm}$. Again, the purpose of this research is to demonstrate how a BER metric could be implemented within the weather cube methodology to quantify the effects of turbulence/scintillation on a notional lasercom-like system and also gain insights into the structure and effects of the weather layers along the communication path. Modeling more typical FSO communications system parameters would likely change the BER statistics due to scintillation/turbulence, but changes in BER statistics obtained with the system parameters modeled herein can still be used to show that realistic turbulence profiles from NWP can have important weather and climate varying effects on lasercom that are not captured with assumed standard or smoothed profiles of $C_{n}^{2}$. Additionally, the extinction effects on the BER statistics should change little for a modeling study of any laser system at the same wavelength (unless the power levels were such that absorption in the beam could cause a change in the index of refraction of the beam path-not likely at $100 \mathrm{~W}$ from a $1-\mathrm{m}$ aperture at $1550 \mathrm{~nm}$ ). Parameters representative of existing FSO communications laser systems will be used in future BER metrics in weather cubes studies.

\subsection{FSO Propagation Scenario, HPCs, and Computational Efficiency}

Another unique aspect of this study is the application of DoD HPCs. Thunder, an Air Force Research Laboratory HPC, was used to process 2016 weather cubes and generate performance statistics for a full sweep of beam paths using HELEEOS. In order to provide a sufficient volume of atmospheric profiles for a representative sample of possible Earth-to-space beam paths, weather cubes specifications for this study consisted of a $10 \times 10$ deg (latitude and longitude) region centered on the National Capital Region (NCR). Weather cubes were generated using 2016 (four times a day - 0000, 0600, 1200, and 1800 UTC) GFS data, resulting in 1450 realistic atmospheres that consider temporal and spatial variations in atmospheric conditions along FSO paths.

Atmospheric effects on laser communication wavelengths become trivial above $30 \mathrm{~km}$; but for completeness, data were generated for surface to $100 \mathrm{~km}$ at $100 \mathrm{~m}$ intervals. HELEEOS was utilized to evaluate a full 0 deg to 360 deg azimuth at 5-deg increments and 0 deg to 90 deg zenith at 2-deg increments. Performance statistics for five zenith angles are presented in Sec. 3.

Ten thousand Thunder nodes were available for use to parallelize data processing jobs. Weather cube data processing was parsed to all available nodes, and one processing job for each weather cubes slice for each day was assigned to each node. This allowed for 192 days or $\sim 6$ months of weather cubes to be processed simultaneously, taking 7.5 wall-clock $\mathrm{h}$ to complete computations. The total wall-clock time for the entire 2016 data set was $15 \mathrm{~h}$. Postprocessing scripts merged the slices together to produce a complete weather cube. HELEEOS was run repeatedly with volumetric environmental inputs from the weather cubes to process background noise and irradiance on the sensor. A similar strategy was used to parallelize HELEEOS computations for 36 azimuthal angle and 30 zenith angle points. Each node ran 36 copies of HELEEOS, which resulted in 360,000 simultaneous calculations and 2 wall-clock $\mathrm{h}$ to complete all computations.

\section{Results}

\subsection{Bit Error Rate Analysis}

This section discusses the performance results of FSO communications in terms of BER. Performance binning statistics are presented for five zenith angles, considering all azimuthal variations. Figure 4 displays BER results for January 7, 2016, at 1200 UTC (0800 local time) for each azimuth and zenith angle on the dome. The red and green colors correspond with high and low BER, respectively.

High BERs, or poor performance, were evident at high zenith (low-elevation) angles throughout the entire data set. This is to be expected since the laser and hence the optical link traverse large amounts of atmosphere where atmospheric attenuation proves troublesome for FSO wavelengths. Such shallow propagation creates a maximum slant path from Earth to space. 




Fig. 4 Weather cube, centered on the NCR, displaying BER by azimuth and zenith angles on January 7, 2016, at 1200 UTC. The color scale indicates high (red) and low (green) BERs. The white arrows correspond to the five zenith angles evaluated for 2016 FSO performance binning.

Large portions of such uplinks cross through the boundary layer, the densest portion of the Earth's atmosphere where molecular and particulate extinction are greatest, thus increasing the probability the signal at the receiver is too weak. Additionally, optical turbulence is greater near the surface of the Earth and plays a significant role in high BERs at low-elevation angles.

Distinct BER layering is another notable feature in the BER dome analysis. This is partially due to variations in the strength of optical turbulence and cloud layers encountered along the path. As anticipated, the 0-deg zenith angle or 90-deg elevation angle was generally proven to be a robust view angle, with low BERs occurring a high percentage of the time. But investigating the BER-layering feature for all days, times, and azimuths offered an optimal performance layer for zenith angles of $20 \mathrm{deg}$ to $40 \mathrm{deg}$. Figure 5 shows an example instance on February 1, 2016, at 0000 UTC when high BERs dominated most elevation angles due to the presence of clouds and precipitation. Note that there remains a region where low BER is observed.

Performance binning analyses were conducted for the following zenith angles, considering all azimuths: $0 \mathrm{deg}, 20 \mathrm{deg}$, $40 \mathrm{deg}, 60 \mathrm{deg}$, and $80 \mathrm{deg}$ zenith angles. Ten performance bins were chosen for this study, and all resulting BER values for the five zenith angles were used to generate histograms and probability distribution functions to demonstrate the frequency that a given BER occurred. Additionally, the sum of all probabilities per zenith angle is equal to one.

\section{February 20160000 UTC (2000L)}

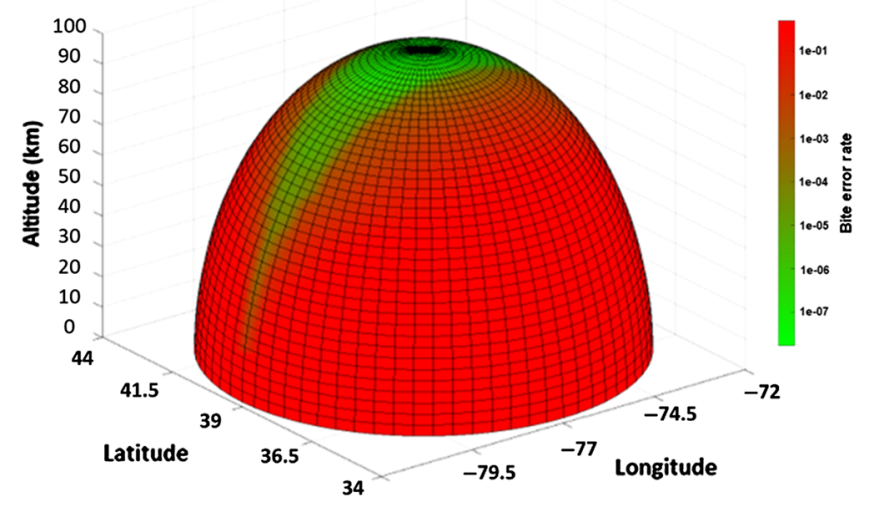

Fig. 5 February 1, 2016, 0000 UTC weather cube displaying BER by azimuth and zenith angles. Note that high BERs (red) across the dome are due to cloud cover. 


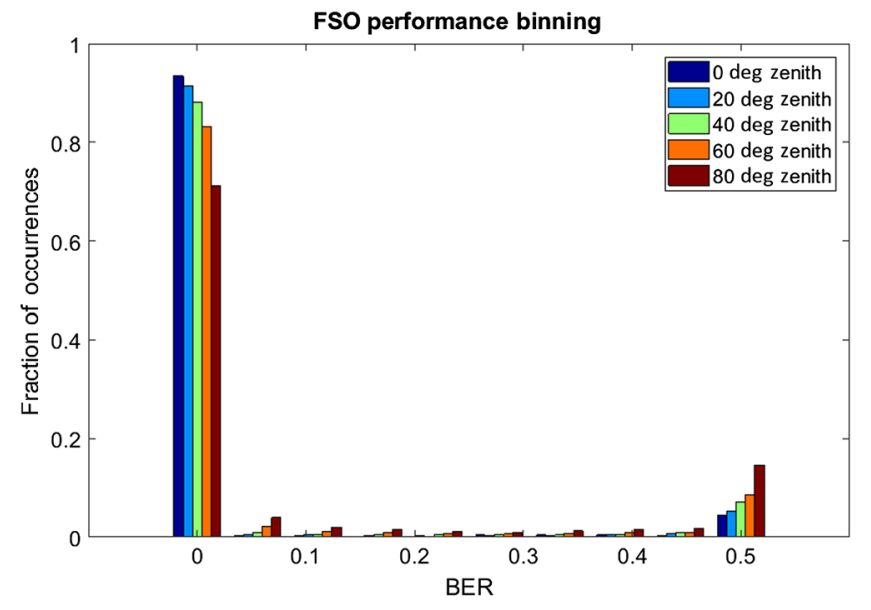

Fig. 62016 FSO performance binning showing the probability that a BER will occur at each zenith angle. The " 0 " BER bin contains all BER occurrences $<1 \times 10^{-6}$.

Figure 6 shows the probability that a given elevation angle resulted in a given BER performance bin. Note that the performance bin denoted as a BER of zero comprised of values that were approximately zero (BER $<1 \times 10^{-6}$ ). A BER of 0.5 was indicated as very poor or unacceptable communication performance, but binning for this category and any BER category above $1 \times 10^{-6}$ still provided useful information about the environmental conditions not necessarily discernible with the typical laser performance metric. The 0-deg zenith angle showed that 93.5\% of occurrences provided very low BERs. There was a direct correlation with an increase in BER with an increase in zenith angle, as expected. Also note that the 80-deg zenith angle fell within the highest BER (0.5) performance bin $15 \%$ of the time, furthering the assumption that low-elevation angles are not reliable for FSO communication. Further investigation was conducted based on BER standards defined by the international telecommunication union (ITU). The ITU suggested a BER of $1 \times 10^{-6}$ or less is acceptable for reliable FSO communication. ${ }^{21}$ Therefore, performance binning analysis was generated for all BER that were equal to or $<1 \times 10^{-6}$, as seen in Fig. 7. An anomaly is seen for the 80 zenith. As mentioned earlier, signal strengths at the receiver for high zenith angle, uplink scenarios are highly attenuated due to molecular and particulate extinction, as well as optical turbulence effects. Figure 8 lends more insight by showcasing the frequency of occurrences per zenith angle. There was a total of 54,010 data points or occurrences in the performance binning analysis when applying

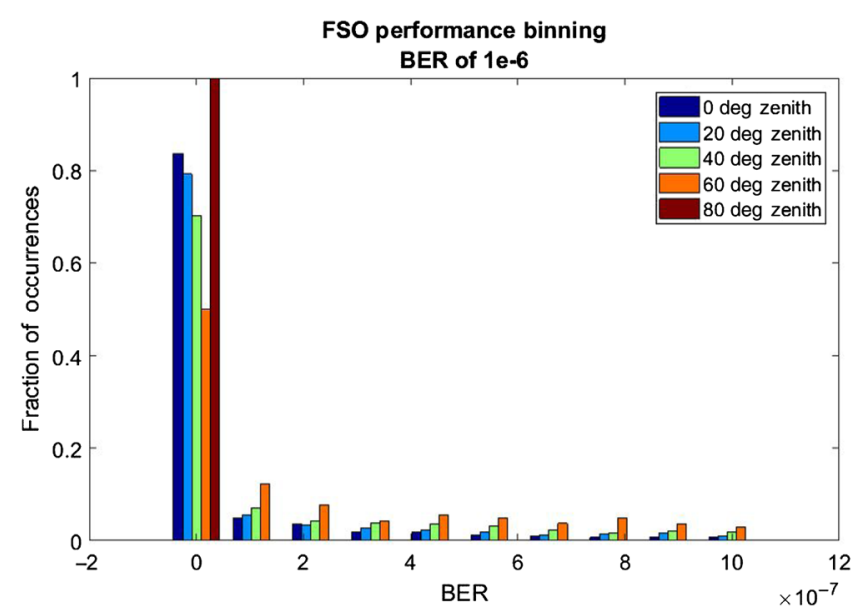

Fig. 72016 FSO performance binning showing the probability that a BER of $1 \times 10^{-6}$ or less will occur at each zenith angle. 




Fig. 8 FSO performance binning displaying the number of occurrences that a BER of $1 \times 10^{-6}$ or less will occur at each zenith angle.

the BER threshold of $1 \times 10^{-6}$. When focusing solely on the lowest BER bin (far left bin), the 80 -deg zenith angle has a $100 \%$ probability that all BERs fall within the lowest (best performance) BER bin, but this only occurred $1.29 \%$ of the time (695 occurrences) within the 2016 data set. Although this high probability appears promising to yield acceptable BERs, the frequency of occurrence is very low and cannot be relied upon for consistency. More analysis is necessary to explain the cause of this feature; however, it appears to suggest that, in general, BER is high for the 80-deg zenith angle; but on rare occasions, atmospheric conditions allow for very good, low BER, FSO communications at high zenith angles.

Figure 7 shows the 0-deg zenith angle scenario yielding optimal FSO communications uplink performance, with an $84 \%$ probability that the BER will be well below the standard. Interestingly, according to Fig. 7, this only occurs 504 times in the data. However, this "artifact" is explainable. It is due to lack of azimuthal variations for the 0-deg zenith angle. Uplink scenarios with zenith angles of $20 \mathrm{deg}$ and $40 \mathrm{deg}$ also show good performance. This becomes most evident when noting the number of occurrences that these two zenith angles fall within the lowest BER performance bin. Approximately half of the occurrences of a very low BER are attributed to the 20-deg zenith angle.

The BER data set was further interrogated to determine azimuthal variations in performance. Figure 9 displays BER at four zenith angles and for 18 azimuth sectors. Each sector consists of 20-deg azimuth angles, as seen in Table 2. For example, sector $1-0$ deg to 20 deg azimuth-is representative of looking north from the NCR; sector 10-180 deg to 200 deg azimuthsimulates uplinks pointing south. Most data fall within the far left, best BER performance bin, and minimal variations in BER per azimuth sector are evident in the 20-deg zenith angle, as seen in Fig. 9(a). A slight performance advantage is noted for sectors 9 to 11 when one's uplink is at 20-deg zenith angle.

Optimal quadrants become more evident as the zenith angle increases. Figure 9(b) shows that north to southeast orientations for FSO communications provide the highest probability of low BERs; whereas, sectors 9 to 10 display a notable drop in the performance for propagating paths oriented toward the south of the NCR. Figures 9(c) to 9(d) show that north to northeastern orientations are highly probable for the highest occurrences of low BER. A significant drop in FSO performance is apparent for sectors 14 to 18 , or 260 deg to 360 deg azimuths, within the lowest (far left) BER performance bin. This is reinforced by an increase in the probability that a high BER will occur for the same orientations by analyzing the 0.5 BER performance bin. Figure 10 provides more insight into an azimuthal dependency on FSO performance when evaluating BER $<1 \times 10^{-6}$. North to northeastern orientations become increasing more reliable as the zenith angle increases. Overall, any orientation for the 20-deg zenith angle offers the same BER results. Figures 10 (b)-(d) show that low BERs are more likely to occur within sectors 1 to 4 . 
FSO performance binning per azimuth sector

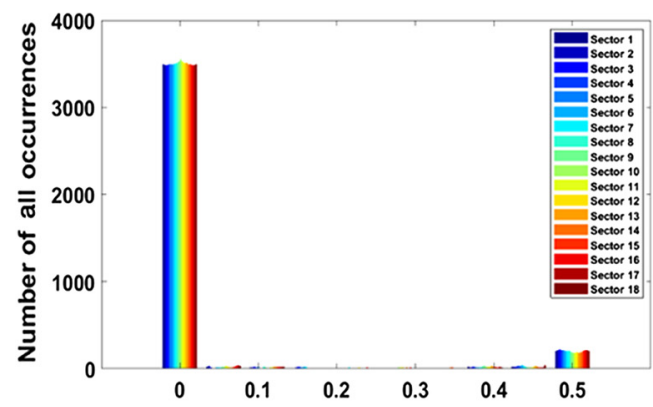

(a)

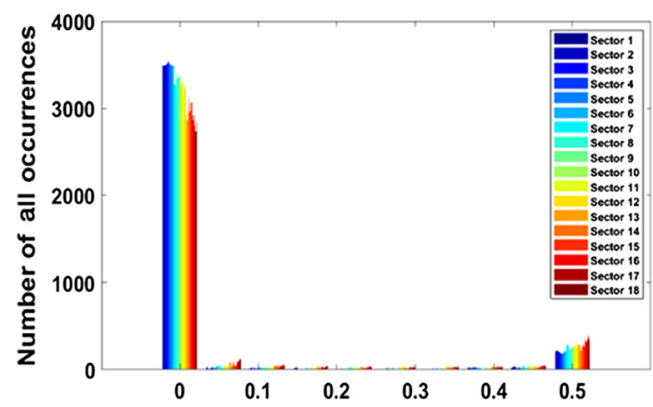

(c)

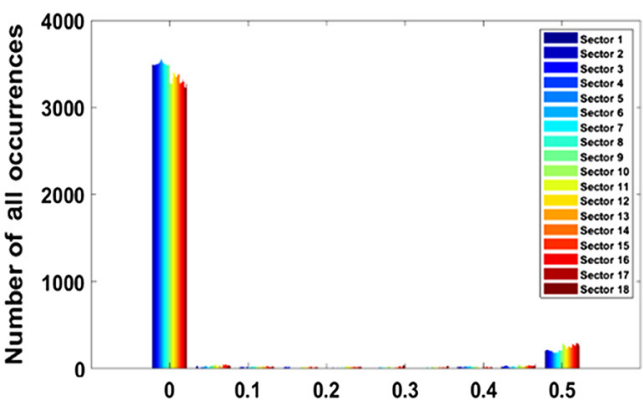

(b)

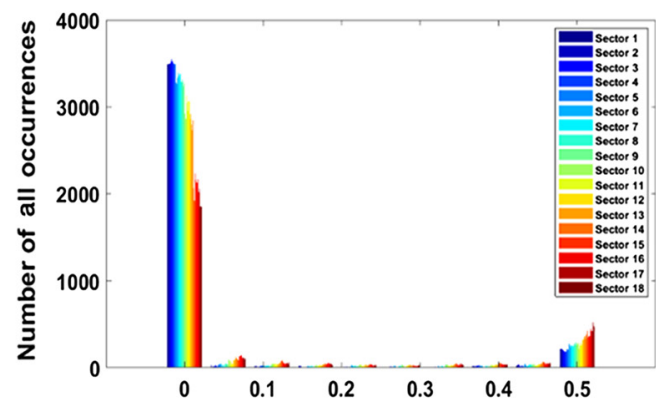

(d)

Fig. 9 Azimuthal variations in 2016 FSO performance binning for (a) 20-deg, (b) 40-deg, (c) 60-deg, and (d) 80-deg zenith angles.

Table 2 Azimuth angles per performance sector.

\begin{tabular}{lccc}
\hline \hline Performance sector & Azimuth (deg) & Performance sector & Azimuth (deg) \\
\hline Sector 1 & 0 to 20 & Sector 10 & 180 to 200 \\
Sector 2 & 20 to 40 & Sector 11 & 200 to 220 \\
Sector 3 & 40 to 60 & Sector 12 & 220 to 240 \\
Sector 4 & 60 to 80 & Sector 13 & 240 to 260 \\
Sector 5 & 80 to 100 & Sector 14 & 260 to 280 \\
Sector 6 & 100 to 120 & Sector 15 & 280 to 300 \\
Sector 7 & 120 to 140 & Sector 16 & 300 to 320 \\
Sector 8 & 140 to 160 & Sector 17 & 320 to 340 \\
Sector 9 & 160 to 180 & Sector 18 & 340 to 360 \\
\hline \hline
\end{tabular}

\subsection{Cloud-Free Line-of-Sight}

The presence of clouds is a critically important parameter for FSO communications. Therefore, understanding the probability that a cloud will inhibit Earth-to-space uplink paths at a given location is necessary to determine the plausibility of FSO communications and the frequency of supplemental MW backup links. Existing CFLOS climatologies are available from the 14th Weather Squadron, formerly known as Air Force Combat Climatology Center, and provide ground-to-space probabilities but do not account for elevation and azimuthal variations. This ground-based, climatological database is available for 415 sites considering two seasons (winter and summer, based on January and July data, respectively) and for view angles of 


\section{FSO performance binning per azimuth sector}

\section{$B E R<1 e-6$}

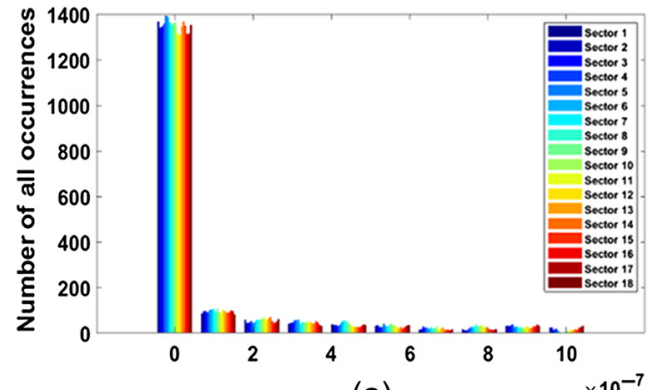

(a)

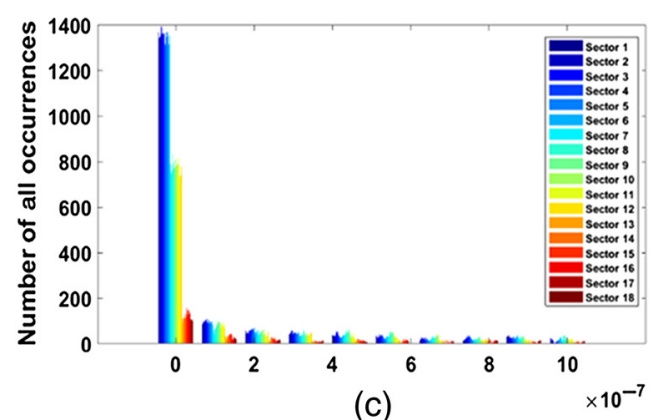



(b)

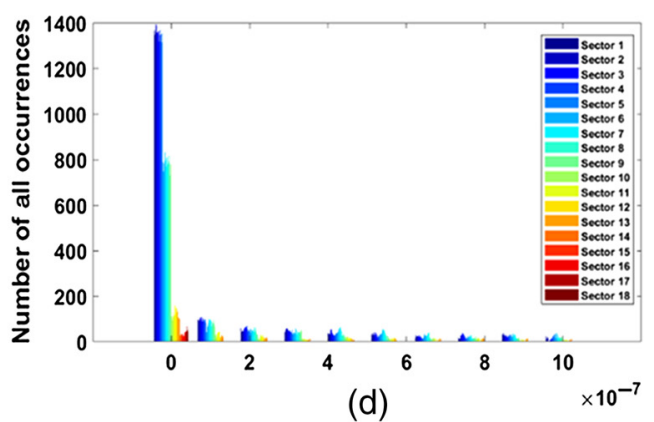

Fig. 10 Azimuthal variations in 2016 FSO performance binning of BER of $1 \times 10^{-6}$ or less for (a) 20-deg, (b) 40-deg, (c) 60-deg, and (d) 80-deg zenith angles.

0 deg to 80 deg zenith. The assumption that the probability of a CFLOS (PCFLOS) for a datavoid region is similar to the assumption of the climatology of a nearby CFLOS site can lead to very inaccurate representations of weather impacts, as sky conditions can vary drastically over short distances.

As previously discussed, recent weather cube enhancements include the implementation of NWP-inferred cloud fields and the development of a cloud model that consequently can be utilized for PCFLOS studies. Monte Carlo simulations considering variations of platform (uplink source) and uplink detector altitudes, as well as slant ranges, can be applied to thousands of different cloudy sky realizations using years of weather cube sky characterizations. This method yields azimuthally dependent PCFLOS statistics for any location worldwide at any time of day, any altitude of interest, and for any view angle, which is a vast improvement over the current CFLOS database- a surface-to-12-km climatology comprised of 415 land sites which does not include azimuthal variations. Weather cubes were generated for January and July 2015, at four times a day, centered on the NCR. Monte Carlo simulations were used to analyze the resultant 248 weather cubes to determine if lines of sight were cloud-free. The uplink-to-LEO simulation considered eight platform heights and a 2-deg azimuthal resolution. Resulting PCFLOS analysis showed seasonal and temporal variations; January and July data represented winter and summer seasons, respectively. Daytime hours consisted of 1200 and 1800 UTC, and 0000 and 0600 UTC were considered nighttime hours. Although the sample set was small, Fig. 11 displays the results at four platform heights for two seasons. Note that these demonstrate the advantages of this tool over the current database available in that the NCR azimuthally dependent PCFLOS analysis shows lower year-round PCFLOS at low-elevation angles toward the west-southwest to westnorthwest and significantly higher CFLOS at low-elevation angles to the northeast in summer. These differences in directional PCFLOS are attributed to greater cloudiness due to the higher elevations of the Appalachian Mountains to the west and northwest of the NCR and decreased cloudiness to the east in summer due to the relative coolness of the Atlantic Ocean. Furthermore, the azimuthal PCFLOS analysis seen in Fig. 11 is fully consistent with the sectional BER analysis in Figs. 9 and 10. 

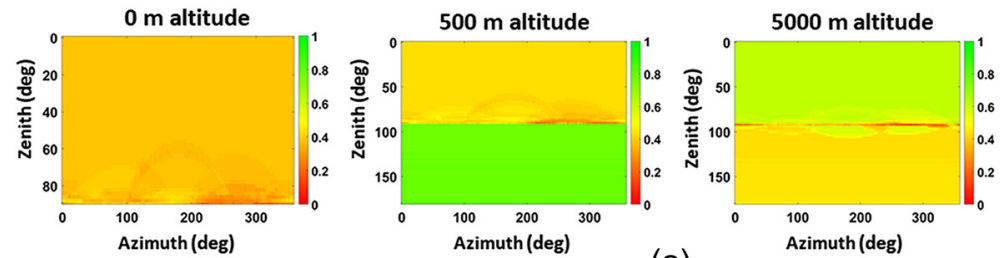

(a)
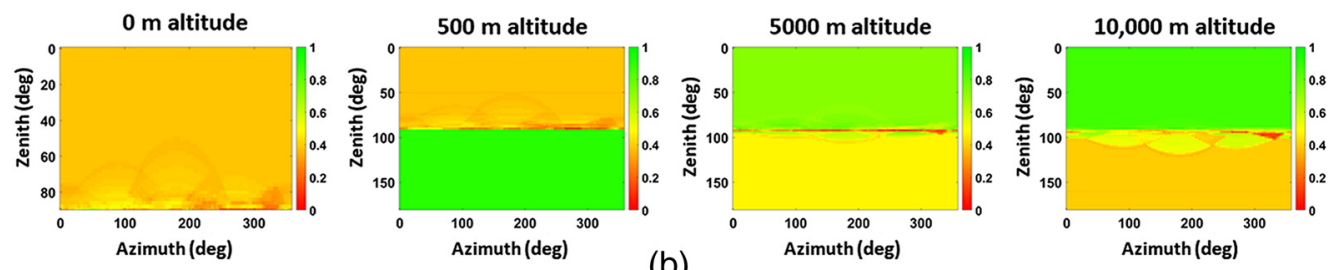

(b)

Fig. 11 Daytime, seasonal PCFLOS with platform altitude variations at the NCRs. Plots are for (a) winter and (b) summer during daylight hours at various altitudes (10, 500, 5000, and 10,000 m) and display all zenith angles ( 0 deg up to 180 deg down) and all azimuthal angles ( 0 deg to $360 \mathrm{deg})$.

A weather cube PCFLOS analysis for the period 2014 to 2017 was also conducted for Holloman AFB, NM, to further investigate orographic effects on PCFLOS analyses. Holloman AFB sits in a north-south oriented valley just east of White Sands missile range. Figure 12 displays PCFLOS for Holloman at the surface, 1000 and 20,000 m elevations above ground level (AGL). At all three heights, it is clearly evident that the higher elevations to the west and east create uplift from the predominantly westerly winds, which, in turn, creates more cloudiness over the mountains and reduces the PCFLOS to the west and east of Holloman AFB. This again illustrates the utility of azimuthally dependent PCFLOS analyses.

Data from the 557th Weather Wing's Worldwide Merged Cloud Analysis (WWMCA) was also leveraged to validate the preliminary weather cube PCFLOS study. WWMCA utilizes analysis of data from multiple environmental satellites, conventional surface observations, and other supporting databases. These include the NOAA Polar Orbiting Environmental satellites, Defense Meteorological Satellite Program satellites, the geostationary orbiting satellites, the Japanese Meteorological Satellite, and the European Space Agency's METEOSATs. ${ }^{23}$ Cloud information is available for up to four cloud layers and 38 cloud parameters per file at a quarter-degree ( $\sim 22.5 \mathrm{~km}$ grid spacing) resolution dating back $\sim 40$ years. WWMCA cloud top height, cloud base height, and total cloud cover were used for PCFLOS analyses. Figure 13 shows PCFLOS generated from weather cubes and WWMCA for a platform altitude of $0 \mathrm{~m}$ at the NCR for both winter and summer seasons. Differences in PCFLOS values at 60-deg zenith and 200-deg azimuth were only $9 \%$ for this small sample set. Future research will be focused on expanding the validation study to $10+$ full years of weather cube analyses. ${ }^{15}$

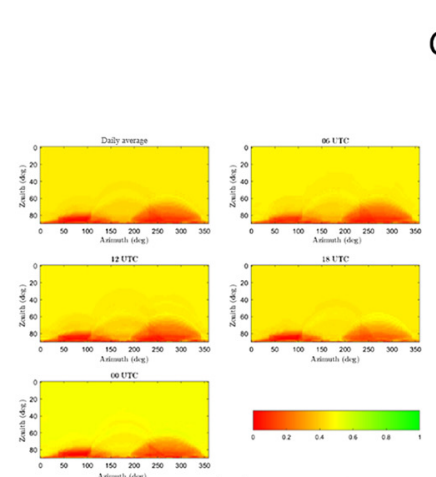

(a)

\section{Cloud-free line-of-sight probabilities Holloman air force base, NM 2014-2017 GFS}

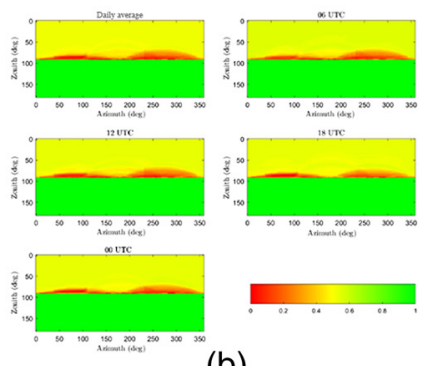

(b)
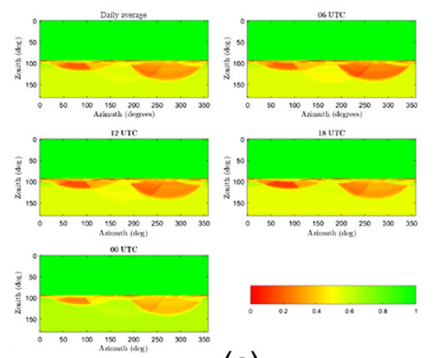

(c)

Fig. 12 PCFLOS for Holloman Air Force Base, NM at surface (a) 0-m, (b) 1000-m, and (c) 20,000-m elevations. 



Fig. 13 PCFLOS generated from GFS and WWMCA data for the NCR at 0-m AGL.

\section{Conclusions}

The primary focus of this research is to apply and demonstrate 4-D weather cubes toward the evaluation of FSO communications performance as related to real-world weather conditions. In order to do so and further demonstrate the tractability of this new tool, the weather cubes were coupled to HELEEOS, a standard scaling law laser propagation code. Large data files were interrogated leveraging HPCs. The results from this study are preliminary and conservative, evaluating only 1 year of FSO communications statistics using a notional, somewhat unrealistic uplink configuration. A prototype BER was selected as the performance metric, and performance binning was accomplished at the NCR as a function of azimuth/zenith angle under various atmospheric conditions. As expected, lower zenith angles were associated with lower BERs. Of note, high BER quantifications not of use to communications did provide useful information about cloud layering and effects at high zenith angles that would be difficult to elucidate using a typical laser performance metric such as peak irradiance at the target. Additionally, azimuthally dependent analyses showed optimal orientations for higher zenith angles were evident for northto-northeastern uplink paths. This study also demonstrated a means to quantify in advance how often FSO communication uplinks in a particular location may need to be supplemented with an MW backup link.

The aggregated, comparative BER binning analyses for the NCR for different times of day and seasons using a full year of data provided numerous occasions of clouds, fog, and precipitation events, thus demonstrating the relevance of 4-D weather cubes for adroit management of CFLOS opportunities to enhance performance analyses of point-to-point as well as evolving multilayer wireless network concepts. Both the NCR and Holloman AFB azimuthally dependent PCFLOS analyses showed lower year-round and seasonal PCFLOS at high zenith angles in certain directions based on regional topography not being captured in any other available CFLOS databases.

Future work will include more analysis to evaluate a longer time period. Up to 10 years of 4-D weather cubes, PCFLOS, and thus BER performance binning will be generated for both FSO and millimeter wave uplinks for a number of worldwide regions. ${ }^{24}$ 


\section{Acknowledgments}

This work was supported by the DoD High Performance Computing Modernization Program (HPCMP) and their Workforce Development HPC Internship Program (HIP) initiative. The authors would like to acknowledge Josiah Bills, a 2018 HIP intern, for his significant contributions to this study. The views expressed in this paper are those of the authors and do not necessarily reflect the official policy or position of the Air Force, the Department of Defense or the U.S. Government.

\section{References}

1. R. Barrios and F. Dios, "Wireless optical communications through the turbulent atmosphere: a review," in Optical Communications Systems, N. Das, Ed., pp. 1-40, IntechOpen, Rijeka, Croatia (2012).

2. J. E. McCrae, S. R. Bose-Pillai, and S. T. Fiorino, "Estimation of turbulence from time-lapse imagery," Opt. Eng. 56(7), 071504. (2017).

3. S. Basu et al., "Estimation of temporal variations in path-averaged atmospheric refractive index gradient from time-lapse imagery," Opt. Eng. 55(9) 090503 (2016).

4. S. Basu et al., "Comparison of the path-weighted $C_{n}^{2}$ from time-lapse imagery and weather radar," in IEEE Aerosp. Conf., Big Sky, Montana, pp. 1-11 (2016).

5. V. I. Tatarskii, "The effects of the turbulent atmosphere on wave propagation," NOAA Report TT-68-50464, US Department of Commerce, Springfield, VA (1971) as translated from author's original Russian publications, "Wave propagation in a turbulent atmosphere," 1967, and "The propagation of short waves in a medium with random inhomogeneities in the approximation of a Markov random process," (1970).

6. A. S. Monin and A. M. Obukhov, "Basic law of turbulent mixing near the ground," Trans. Akad. Nauk. 24(151), 163-187 (1954).

7. J. C. Wyngaard, "On surface layer turbulence," in Workshop on Micrometeorol, D. A. Haugen, Ed., pp. 101-148, American Meteorological Society (1973).

8. D. Sadot and N. S. Kopeika, "Forecasting optical turbulence strength on the basis of macroscale meteorology and aerosols: models and validation," Opt. Eng. 31(2), 200-212 (1992).

9. S. Bendersky, N. S. Kopeika, and N. Blaunstein, "Atmospheric optical turbulence over land in Middle East coastal environments: prediction modeling and measurements," Appl. Opt. 43(20), 4070-4079 (2004).

10. D. Sadot and N. S. Kopeika, "Forecasting optical turbulence strength: effects of macroscale meteorology and aerosols," Proc. SPIE 1442, 324-334 (1991).

11. A. A. B. Raj, J. Selvi, and S. Durairaj, "Comparison of different models for ground-level atmospheric turbulence strength $\left(C_{n}^{2}\right)$ prediction with a new model according to local weather data for FSO applications," Appl. Opt. 54(4), 802-815 (2015)

12. J. Latal et al., "Regression models utilization for RSSI prediction of professional FSO link with regards to atmosphere phenomena," in Int. Conf. Broadband Commun. for Next Gener. Networks and Multimedia Appl. (CoBCom), Graz (2016).

13. I. I. Kim, B. McArthur, and E. Korevaar, "Comparison of laser beam propagation at $785 \mathrm{~nm}$ and $1150 \mathrm{~nm}$ in fog and haze for optical wireless communications," Proc. SPIE 4214, 26-37 (2001).

14. S. T. Fiorino et al., "Validation of a UV-to-RF high-spectral-resolution atmospheric boundary layer characterization tool" J. Appl. Meteorol. Climatol. 53, 136-156 (2014).

15. J. E. Schmidt et al., "4D weather cubes and defense applications," in Defense Innovation Handbook, A. B. Badiru and C. Barlow, Eds., pp. 257-279, CRC Press, Boca Raton, Florida (2018).

16. J. Toth et al., "Classification prediction analysis of RSSI parameter in hard switching process for FSO/RF systems," Measurement 116, 602-610 (2018).

17. J. L. Burley et al., "A fast two-stream-like multiple scattering method for atmospheric characterization and radiative transfer," J. Appl. Meteorol. Climatol. 56, 3049-3063 (2017). 
18. G. K. Rutledge, J. Alpert, and W. Ebuisaki, "NOMADS: a climate and weather model archive at the National Oceanic and Atmospheric Administration," Bull. Am. Meteorol. Soc. 87, 327-342 (2006).

19. J. O. Kondo, O. Kanechika, and N. Yasuda, "Heat and momentum transfers under strong stability in the atmospheric surface layer," J. Atmos. Sci. 35, 1012-1021 (1978).

20. S. T. Fiorino, "Satellite and radar measurement of CT2, Cn2, and Cv2," in Imaging and Appl. Opt. 2014, OSA Tech. Digest, Optical Society of America, p. PM1E.1 (2014).

21. R. J. Alliss and B. D. Felton, "Validation of optical turbulence simulations from a numerical weather prediction model in support of adaptive optics design," in Proc. Adv. Maui Opt. and Space Surveill. Technol. Conf., S. Ryan, Ed., p. E54, The Maui Economic Development Board., Wailea, Hawaii (2009).

22. L. C. Andrews and R. L. Phillips, Laser Beam Propagation Through Random Media, SPIE Press, Bellingham, Washington (2005).

23. J. L. Burley et al., "A remote sensing and atmospheric correction tool for assessing multispectral radiative transfer through realistic atmospheres and clouds" J. Atmos. Ocean. Technol. 36, 203-216 (2018).

24. S. T. Fiorino et al., "Implications of 4-D weather cubes for improved cloud free line of sight assessments of free space optical communications link performance," Proc. SPIE 10981, 109810S (2019).

Steven T. Fiorino received his BS degrees from Ohio State (1987) and Florida State (1989), an MS degree in atmospheric dynamics (Ohio State, '93), and his $\mathrm{PhD}$ in physical meteorology (Florida State '02). He is a professor of Atmospheric Physics at AFIT and director, AFIT Center for Directed Energy. His research interests include microwave remote sensing, weather signal processing, and atmospheric effects on weapon systems. He is a member of SPIE, OSA, AMS, AIAA, and DEPS.

Santasri R. Bose-Pillai received her BSEE from Jadavpur University, India, in 2000, MSEE in 2005 and PhD in Electrical Engineering in 2008 from New Mexico State University. Currently, she is a research assistant professor at AFIT's Center for Directed Energy within Engineering Physics Department. Her research interests include propagation and imaging through atmosphere, partially coherent sources and laser communications. She is a member of DEPS and a senior member of OSA and SPIE.

Jaclyn Schmidt received her BS degree in meteorology from the University of South Alabama in 2010. She is currently a research meteorologist at AFIT's Center for Directed Energy. Her research interests include numerical weather modeling and enhancements to atmospheric characterization tools to benefit directed energy and intelligence communities. She is a member of DEPS and AMS.

Brannon Elmore received his BS degree in computer science from Wright State University in 2014. He is the lead software developer for AFIT's Center for Directed Energy. His research efforts are focused on the continuous improvement of LEEDR and HELEEOS, and the core applications of the AFIT Directed Energy and Atmospheric Models software package.

Kevin Keefer received his BS degree in atmospheric physics from the United States Air Force Academy in 1981, his MS degree in Systems Management from the University of Southern California in 1983, engineering physics from AFIT in 1985, and his $\mathrm{PhD}$ in solid state physics from AFIT in 1990. Currently, he is a research physicist at AFIT's Center for Directed Energy. His research interests include microphysical and radiative effects associated with atmospheric molecular and aerosol constituents and turbulence. 